\title{
Phytotoxic effect of silver nanoparticles in Triticum aestivum: Improper regulation of photosystem $I$ activity as the reason for oxidative damage in the chloroplast
}

\author{
A. RASTOGI ${ }^{*+,}$, M. ZIVCAK ${ }^{* *}$, D.K. TRIPATHI ${ }^{* * *}$, S. YADAV $^{* * * *}$, H.M. KALAJI ${ }^{\#, \#,}$ \\ and M. BRESTIC ${ }^{* *,+}$
}

Department of Meteorology, Poznan University of Life Sciences, Piatkowska 94, 60-649 Poznan, Poland* Department of Plant Physiology, Slovak University of Agriculture, A. Hlinku 2, 94976 Nitra, Slovak Republic** Amity Institute of Organic Agriculture (AIOA)Amity University, Noida Sector 125, Noida, Uttar Pradesh- 201313, India ${ }^{* * *}$

Department of Biotechnology, Hemvati Nandan Bahuguna Garhwal (Central) University, Srinagar Garhwal, Uttarakhand, 246174, India****

Institute of Technology and Life Sciences (ITP), Falenty, Al. Hrabska 3, 05-090 Raszyn, Poland ${ }^{\#}$

White Hill Company, Żurawia 71/3, 15-540 Białystok, Poland ${ }^{\# \#}$

\begin{abstract}
Silver nanoparticles (AgNPs) are used in several industries and their continuous release into environment can damage the ecosystem. Our study observed the impact of two concentrations of AgNPs (1 and $5 \mathrm{mM})$ on wheat seedlings. In this study, the treatment of AgNPs was found to have a significant impact on growth parameters, such as root and shoot length of wheat seedlings, in addition to that biochemical parameters, such as activity of catalase, glutathione, flavonoid, and chlorophyll concentrations, were also affected. AgNPs apparently suppressed the photosynthetic activity of the seedlings, with a clear destruction of photosystems at $5 \mathrm{mM}$ concentration. The result also indicated an improper regulation of PSI electron transport, resulting in a damage to chloroplast structure, including photosystem itself. Our study presents a mechanism of the AgNPs action of on photosynthetic processes in plants.
\end{abstract}

Additional key words: antioxidants; chlorophyll fluorescence; JIP test.

\section{Introduction}

Nanoparticles cover a heterogeneous range of materials (Santos et al. 2015), but only a few of them are extensively used. The present environment is at risk to be exposed by extensively used nanoparticles. Silver nanoparticles (AgNPs) are among one of the most used nanoparticles, which can be found in various products. AgNPs can be used as antimicrobial agents, waste-water treatment agent, shampoo, toothpaste, soap, food-packaging materials, food-storage containers, room sprays, detergents, fabrics, paint, etc. (Boxall et al. 2007, Rai et al. 2009, Wijnhoven et al. 2009). Recent reports have shown that about $6 \%$ of
AgNPs produced in the United States ends up in sludge, whereas around 3.5\% in surface water; thus, AgNPs may contaminate agricultural lands (Khaydarov et al. 2009, ElTemsah and Joner 2012).

Previous studies have shown that AgNPs may interfere with plant metabolism and may have a positive or negative impact, which depends on its concentration, size, and properties (Gruyer et al. 2014, Pallavi et al. 2016, Jasim et al. 2017, Rastogi et al. 2017). Dimkpa et al. (2013), have shown that AgNPs of 10-nm size, when hydroponically applied at a concentration of $2.5 \mathrm{mg} \mathrm{kg}^{-1}$, are nontoxic for wheat, whereas, when present in excess they causes a toxic response in the form of morphological variation and

Received 4 March 2018, accepted 19 July 2018.

${ }^{+}$Corresponding authors; phone: 0048-53 51 49922, e-mail: anshu.rastogi@up.poznan.pl (A. Rastogi); phone: 00421 - 376144448 , e-mail: marian.brestic@uniag.sk (M. Brestic)

Abbreviations: ABS - absorption; AgNPs - silver nanoparticles; UV - ultra-violet radiation; FLAV - flavonoids; GSH - glutathione; CAT - catalase; OEC - oxygen-evolving complex; $\mathrm{OEC}_{\mathrm{act}}$ - active oxygen-evolving complex; SSA - 5-sulfosalicylic acid; $\mathrm{F}_{\mathrm{m}}$ maximum fluorescence intensity; NPQ - nonphotochemical quenching; $\mathrm{F}_{\mathrm{v}} / \mathrm{F}_{\mathrm{m}}$ - photosynthetic efficiency of PSII under dark adaptation; $\mathrm{P}_{\mathrm{m}}$ - maximum amplitude of P700 signal; $\Phi_{\mathrm{PSII}}-$ maximum quantum yield of PSII photochemistry; $\Phi_{\mathrm{NPQ}}-$ quantum yield of regulated nonphotochemical energy loss in PSII; $\Phi_{\mathrm{NO}}$ - quantum yield of nonregulated nonphotochemical energy loss in PSII; $\Phi_{\mathrm{PSI}}-$ maximum quantum yield of PSI; $\Phi_{\mathrm{ND}}$ - quantum yield of regulated nonphotochemical energy loss in PSI; $\Phi_{\mathrm{NA}}-$ quantum yield of nonregulated nonphotochemical energy loss in PSI; $\Phi_{\mathrm{P}_{0}}$ - photochemical efficiency; $\mathrm{PI}_{\mathrm{abs}}$ - overall photochemical performance; ROS - reactive oxygen species; $\Psi_{\mathrm{ETo}}-$ electron transport at PSII acceptor side; RC/ABS - fraction of active PSII reaction centers.

Acknowledgement: This work was supported by the research project of the Slovak Research and Development Agency under the project APVV-15-0721, VEGA 1/0923/16, and National Science Centre Poland, under the project UMO-2016/21/B/ST10/02271. AR thanks to Slovak Academic Information Agency (SAIA) for providing National Scholarship for research in the year 2017. 
accumulation of oxidized glutathione. The AgNp of $10 \mathrm{~nm}$ was observed to enhance a root length at a concentration of $2.5 \mathrm{mg} \mathrm{L}^{-1}$ in barley, whereas it reduced the root length at a higher concentration (Gruyer et al. 2014). All the studied concentrations of AgNP were observed to reduce the root length when applied on lettuce, whereas for radish, the studied concentrations of AgNP were not able to cause any significant impact (Gruyer et al. 2014). The biosynthesized silver nanoparticles of 8-21 nm diameter at $1 \mu \mathrm{m} \mathrm{mL}^{-1}$ concentration were observed to significantly impact the growth parameters such as root lenght, shoot lenght, and fresh mass of fenugreek seedlings (Jasim et al. 2017). The foliar treatment of wheat seedlings by 35-nm AgNPs was observed to be ineffective in causing positive or negative impact at studied concentrations (Pallavi et al. 2016). Several other studies have indicated that AgNPs of different sizes in variable concentrations can lead to morphological changes in plants, in addition, it can also influence the reduction in root/shoot and overall biomass (Hawthorne et al. 2012, Yin et al. 2012, Mirzajani et al. 2013, Pokhrel and Dubey 2013, Qian et al. 2013, Zuverza-Mena et al. 2016, Tripathi et al. 2017, Vinković et al. 2017). Different studies have shown that the plants exposed to AgNPs produce a higher amount of reactive oxygen species (ROS) and antioxidant molecules (Dimkpa et al. 2013, Jiang et al. 2014, Belava et al. 2017, Tripathi et al. 2017). Few studies have shown a significant decline in photosynthetic pigment contents as a response to AgNPs exposure (Qian et al. 2013, Tripathi et al. 2017). Whereas, according to our knowledge, only a few studies have been focused on the impact of AgNPs on photosynthetic efficiency of plants.

Photosynthesis is an important, and one of the most complex phenomena in plants. Chlorophyll (Chl) fluorescence emission is a process which is going in parallel with photosynthesis in plants, therefore, photosynthetic process and $\mathrm{Chl}$ fluorescence are closely related. It has been now established, that changes in photosynthetic activity due to different environmental factors can be detected through the observation of the changes in Chl fluorescence (Kalaji et al. 2014). Analysis of Chl fluorescence parameters can be used as a precise tool for testing a direct impact of adverse environmental conditions on photosynthesis and, therefore, the indirect assessment of their impact on plants (Kalaji et al. 2014). Therefore, the study of photosynthetic efficiency is needed to understand the plant-nanoparticle interaction. $\mathrm{CuO}$ nanoparticles were observed to influence photosynthesis in Lemna gibba L. (Perreault et al. 2014). The authors observed a significant difference in Chl $a$ fluorescence, which indicated a decrease in the number of active PSII reaction centers, as a result to $\mathrm{CuO}$ nanoparticles exposure.

Because of its economic importance, wheat was chosen as an experimental crop in this study. The phytotoxicity of AgNPs on wheat seedlings was analyzed by observing variations in morphology, physiology, and contents of biochemical compounds.

\section{Materials and methods}

Silver nanoparticle preparation: The nano-powder of AgNP of particle size $<100 \mathrm{~nm}$ was purchased from $S I G M A-A L D R I C H^{\circledR}$. The nanoparticles were containing polyvinylpyrrolidone (PVP) as a dispersant. The nanoparticles were sterilized for $20 \mathrm{~min}$ under ultraviolet radiation (UV), in laminar flow. The stock of $100 \mathrm{mM}$ AgNP suspension was prepared in sterile distilled water using sterile glassware. The nanoparticle suspension was prepared using the ultrasonic bath for $3 \mathrm{~h}$; the water in the ultrasonic bath was exchanged with the ice-cold water, every $20 \mathrm{~min}$. A homogenously dispersed nanoparticle suspension was obtained. Different dilutions (1 and $5 \mathrm{mM}$ ) of AgNP were prepared with sterile water. Nanoparticle suspension of $20 \mathrm{ml}$ was added to sterile petri plates containing UV-treated filter paper.

Seed preparation and growth conditions: The seeds of spring wheat (Triticum aestivum) cv. "Corso" were obtained from phenotyping laboratory of Slovak Agriculture University in Nitra. Spring genotype was selected because of its fast development and higher initial vitality. The seeds were washed for $1 \mathrm{~h}$ in running tap water; surface sterilized with $0.1 \% \mathrm{HgCl}_{2}$ for 2.5 min with $80 \%$ ethanol, then the seeds were washed three times with sterile distilled water. The seeds were distributed in Petri plates with 0,1 , and $5 \mathrm{mM}$ AgNPs (15 seeds each). The seeds were grown in a plant chamber with $250 \mu$ mol(photon) $\mathrm{m}^{-2} \mathrm{~s}^{-1}$, photoperiod of 16-h light/8-h dark, relative air humidity $60-70 \%$, and day temperature of $22^{\circ} \mathrm{C}$ and $18^{\circ} \mathrm{C}$ at night for three weeks. Three replica plates were kept for each condition.

Plant morphological analysis: The growth of seedlings was observed at regular intervals for their morphological variations. Root and shoot sizes were measured using graph paper and scale. The final measurement for root and shoot sizes were performed after three weeks of the AgNPs treatment. The statistical analysis was done by random measurements of ten plants from three different plates under the same conditions. The root morphology was observed using Nikon Optiphot (Japan) light microscope fitted with Canon (Japan) digital camera.

Catalase and glutathione: Catalase activity (EC 1.11.1.6) and reduced glutathione (GSH) from the wheat root was measured using catalase assay kit (CAT100, SIGMA$A L D R I C H)$ and glutathione assay kit (CS0260, SIGMA$A L D R I C H)$, respectively. For catalase, $0.5 \mathrm{~g}$ of fresh roots were collected and homogenized with $5 \mathrm{~mL}$ of $50 \mathrm{mM}$ potassium phosphate buffer (PBS, pH 7.0) containing $0.4 \%$ PVP in a mortar and pestle on the ice. The homogenate was further centrifuged at $10,000 \times g$ for 20 min at $4^{\circ} \mathrm{C}$. The supernatant was collected and examined for catalase activity following the protocol provided with the kit. The reaction time was kept for 1 min for $10 \mu l$ of the supernatant. The spectroscopic measurement was taken at $240 \mathrm{~nm}$ using Cary $60 \mathrm{UV}$-Vis spectrophotometer (Agilent, Santa Clara, USA). Catalase was calculated according to the kit and expressed as enzyme Unit per gram of fresh mass, where enzyme Unit is amout required for transformation of $1 \mu \mathrm{mol}$ of the hydrogen peroxide per min at standard 
conditions. For the measurement of GSH, the root tissues were frozen dry with liquid nitrogen immediately after excision. The tissues were grounded in a mortar and pestle with liquid nitrogen to obtain a fine powder. Powdered sample of $0.1 \mathrm{~g}$ was taken and mixed with $300 \mu \mathrm{l}$ of $5 \%$ 5 -sulfosalicylic acid (SSA) and vortexed vigorously for $1 \mathrm{~min}$, then $2,100 \mu \mathrm{l}$ of $5 \% \mathrm{SSA}$ was added and homogenized with a 3-ml PTFE pestle in a glass tube for $5 \mathrm{~min}$. The samples were adapted to $4^{\circ} \mathrm{C}$ and centrifuged at $10,000 \times$ $g$ for $10 \mathrm{~min}$ at $4^{\circ} \mathrm{C}$. The further analyses were performed according to the protocol provided with the kit and expressed as nmol per gram of dry mass. The spectroscopic measurement was done at $412 \mathrm{~nm}$ using Cary 60 UV-Vis spectrophotometer (Agilent, Santa Clara, USA). Five repetitions were performed for both antioxidants to calculate standard deviation.

Chl and flavonoid: The Chl content was measured noninvasively by using SPAD-502 (Minolta Camera Co., Osaka, Japan). The Chl meter readings were obtained from the middle of the grown leaves. The flavonoid content was measured noninvasively by using Multiplex-3fluorimetric sensor (Force-A, Orsay, France). The Multiplex measures fluorescence emitted by $\mathrm{Chl}$, in the red and far-red spectral regions, under excitation by light-emitting diodes of $375,450,515$, and $630 \mathrm{~nm}$. The synchronized filteredphotodiode detectors recorded fluorescence in yellow, red, and far-red (Zivcak et al. 2017). The relative amount of flavonoids (FLAV) was calculated observing Chl fluorescence signals under UV (FRF-UV) and red excitation (FRF-R) (Sytar et al. 2015).

The measurements were performed on nine randomly selected plants for each treatment, by taking the average value of three measurements from the same seedling. The measurements were converted to the percentage assuming the mean value of the control to be $100 \%$.

P700 redox state and $\mathrm{Chl}$ fluorescence: $\mathrm{P} 700$ redox state and Chl $a$ fluorescence were simultaneously measured through Dual PAM-100 (Heinz Walz GmbH, Germany). The plants were transferred to laboratory bench and darkadapted for $20 \mathrm{~min}$ in a dark box, and around $2 \mathrm{~min}$ in the measuring head. To activate the photosynthetic processes, a light intensity of $134 \mu \mathrm{mol}$ (photon) $\mathrm{m}^{-2} \mathrm{~s}^{-1}$ (similar to ambient light) was used. Once the steady state was reached, a rapid light curve was triggered [at light intensities of 14, 30, 61, 103, 134, 174, 224, 347, 539, 833, 1,036; 1,295; 1,602; and $1,930 \mu \mathrm{mol}$ (photon) $\mathrm{m}^{-2} \mathrm{~s}^{-1} ; 30 \mathrm{~s}$ at each light intensity] with saturation pulse and far-red pulse. The measurements were performed on three plants for each experimental conditions, and different parameters were calculated as described previously in Brestic et al. (2016).

Chl fluorescence induction curve was measured using Handy PEA fluorometer (Hansatech Instruments Ltd., Norfolk, UK). The plants were transferred to laboratory bench for the measurement, the plants were dark-adapted for $20 \mathrm{~min}$ using the clip provided by the producer. Measurements were performed following the standard protocol with a 3,500 $\mu$ mol(photon) $\mathrm{m}^{-2} \mathrm{~s}^{-1}$ of light intensity (as max) and a 1-s duration of the pulse light (saturation light). Analysis of measured Chl $a$ fluorescence signal was based on a JIP test (Strasser and Strasser 1995, Srivastava et al. 1999, Strasser et al. 2000, 2004, 2010). The measurement was performed in the middle of the oldest leaf from nine plants for a treatment. The Chl induction curve was drawn from the mean of the obtained data points.

\section{Results}

Plant morphology: The wheat seedlings were analyzed for the root and shoot growth each week. The toxic concentration of AgNPs for this study was determined by observing the morphological changes in the seedlings after a week of germination (Fig. 1S, supplement). More than $50 \%$ decrease in root and shoot growth was observed after three weeks of germination (Table 1, Fig. 1A). At $5 \mathrm{mM}$ AgNPs concentration the seedling roots were observed to avoid its contact with filter paper (Fig. 2S, supplement). The microscopic study showed that the roots at $5 \mathrm{mM}$ concentration were darker in comparison to control and a large number of root hairs was observed at $5 \mathrm{mM}$ concentration (Fig. $1 B$ ). The results clearly indicated that the concentrations studied were phytotoxic for wheat.

Table 1. Impact of AgNPs on root and shoot length of wheat after 3-week exposure. The data are presented as mean of at least three measurements with its standard deviation.

\begin{tabular}{lcc}
\hline AgNPs $[\mathrm{mM}]$ & Root $[\mathrm{cm}]$ & Shoot $[\mathrm{cm}]$ \\
\hline 0 & $11.39 \pm 1.22$ & $12.25 \pm 2.06$ \\
1 & $5.54 \pm 0.89$ & $5.2 \pm 0.6$ \\
5 & $4.2 \pm 0.56$ & $4.94 \pm 0.73$ \\
\hline
\end{tabular}

Catalase and glutathione: The catalase activity increased by $50 \%$ in plant roots treated with $1 \mathrm{mM} \mathrm{AgNPs,} \mathrm{whereas}$ it was $100 \%$ higher in roots treated with $5 \mathrm{mM} \mathrm{AgNPs}$ when compared with control plants (Fig. 2A). The GSH content also increased in plant roots by approximately 100 and $300 \%$ at $1 \mathrm{mM}$ and $5 \mathrm{mM}$ AgNPs, respectively (Fig. 2B). The results indicate the increase in catalase activity and in the amount of GSH as the protective molecules in plant roots in response to AgNPs exposure.

Total Chl concentration and flavonoids: The total Chl in wheat decreased by $20 \%$ at both studied concentrations of AgNPs (Fig. 3A). As a response to $1 \mathrm{mM}$ and $5 \mathrm{mM}$ AgNPs exposure, the flavonoid content of the wheat leaves increased by 100 and $200 \%$, respectively (Fig. $3 B$ ). The results indicate the decrease in total $\mathrm{Chl}$ content, whereas a significant increase in flavonoid content as a response to AgNPs exposure of wheat plants.

P700 redox state and $\mathrm{Chl}$ fluorescence: Fig. 4 indicates the response of different fluorescence parameters to $1 \mathrm{mM}$ and $5 \mathrm{mM}$ AgNPs. $\mathrm{P}_{\mathrm{m}}$ indicates the maximum amplitude of P700 signal, which decreased by around 15 and $50 \%$ in $1 \mathrm{mM}$ and $5 \mathrm{mM} \mathrm{AgNPs-treated} \mathrm{plants,}$ respectively, when compared with control (Fig. 4A). 

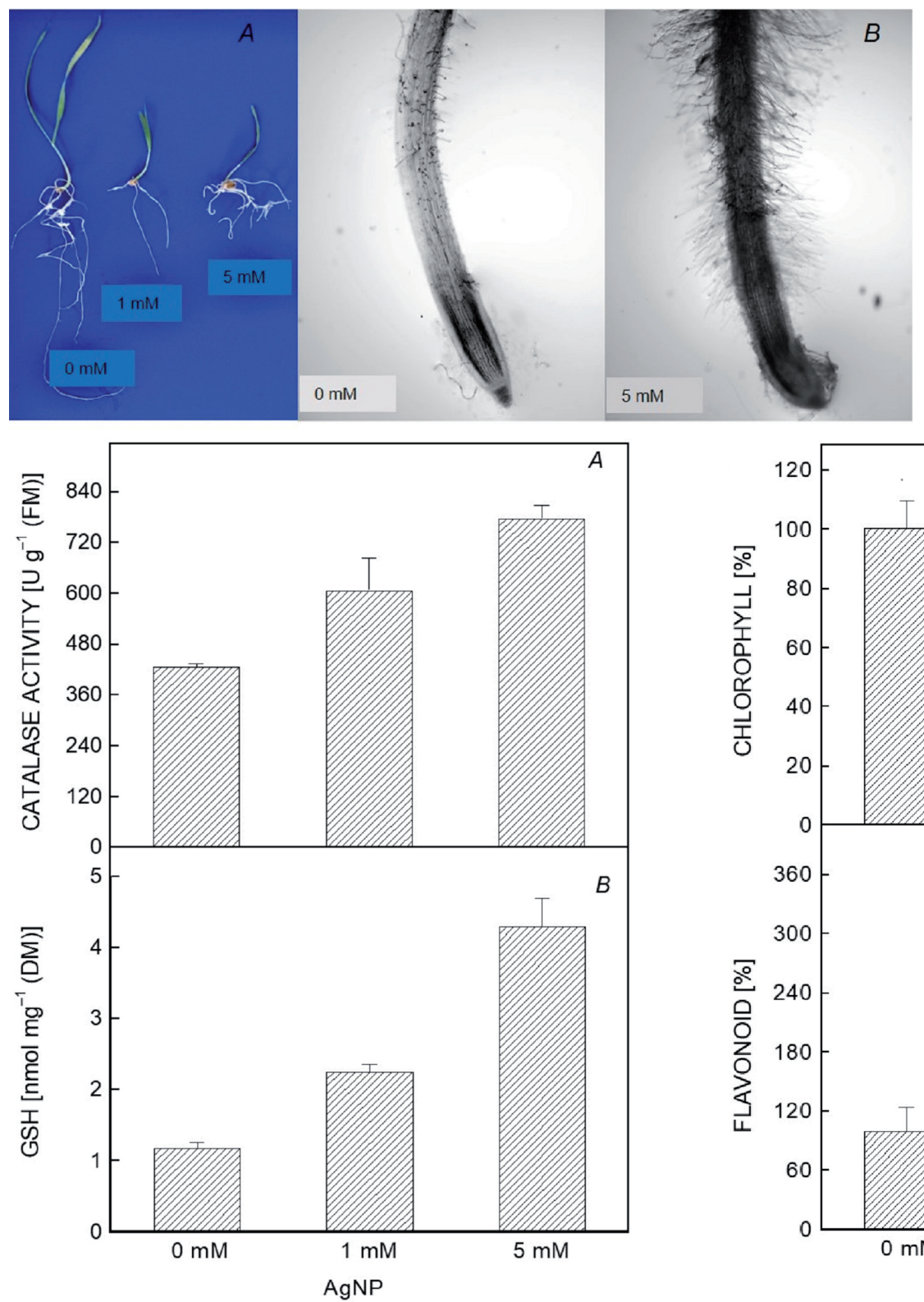

Fig. 2. Impact of AgNPs on antioxidants: $(A)$ The enzymatic activity of catalase when exposed to AgNPs. The measurement was taken from plant root in enzyme Unit per gram fresh mass $\left[\mathrm{U} \mathrm{g}^{-1}(\mathrm{FM})\right]$. (B) The GSH content of the wheat root under different exposure to AgNPs. The amount of GSH was expressed in $\mathrm{nmol} \mathrm{mg}^{-1}(\mathrm{DM})$.

$F_{v} / F_{m}$ indicates the estimated maximum quantum efficiency of PSII photochemistry, which decreased by 12 and $80 \%$, whereas, nonphotochemical quenching (NPQ) decreased by 10 and $85 \%$ in $1 \mathrm{mM}$ and $5 \mathrm{mM}$ AgNPstreated plants, respectively, when compared with control (Fig. 4B,C). $\phi_{\text {PSII }}$ indicates the estimated effective quantum yield of PSII at given PAR, which decreased by 25 and $95 \%$ at 833 PAR for $1 \mathrm{mM}$ and $5 \mathrm{mM}$ AgNPs-treated plants, respectively, when compared with control (Fig. $4 D)$. $\phi_{\mathrm{NPQ}}$ indicates the quantum yield of $\mathrm{pH}$-dependent energy dissipation in PSII, whereas $\phi_{\mathrm{NO}}$ indicates quantum

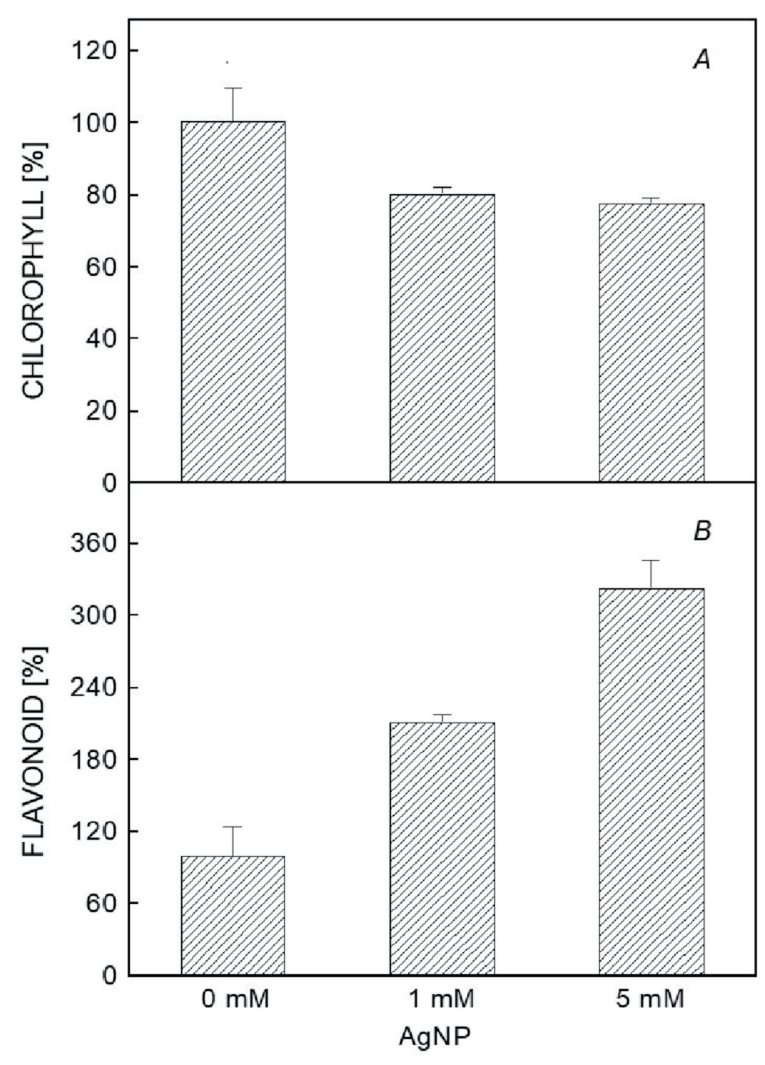

Fig. 3. Relative content of chlorophyll and flavonoids in wheat leaves under different AgNPs exposure conditions.

yield of nonregulated energy dissipation in PSII. At $1 \mathrm{mM}$ AgNPs concentration, only 4\% decrease in $\phi_{\mathrm{NPQ}}$, and $5 \%$ increase in $\phi_{\mathrm{NO}}$ was observed, whereas, the differences were observed to be very significant for these parameters, in case of $5 \mathrm{mM}$ AgNPs, when compared with control (Fig. 4E,F). Parameter $\phi_{\text {PSI }}$ represent the photochemical quantum yield of PSI; it slightly increased in case of $1 \mathrm{mM}$ AgNPs concentration, whereas a significant decrease in $\phi_{\mathrm{PSI}}$ was observed in the plant sample treated with $5 \mathrm{mMAgNPs}$ when compared with control. Parameter $\phi_{\mathrm{ND}}$, indicating the nonphotochemical quantum yield of PSI, which is oxidized at a given state, decreased by 70 and $40 \%$ for $1 \mathrm{mM}$ and $5 \mathrm{mM}$ samples, respectively (Fig. 4H). Parameter $\phi_{\mathrm{NA}}$, which indicates nonphotochemical quantum yield of PSI that cannot be oxidized by a saturation pulse at a given 


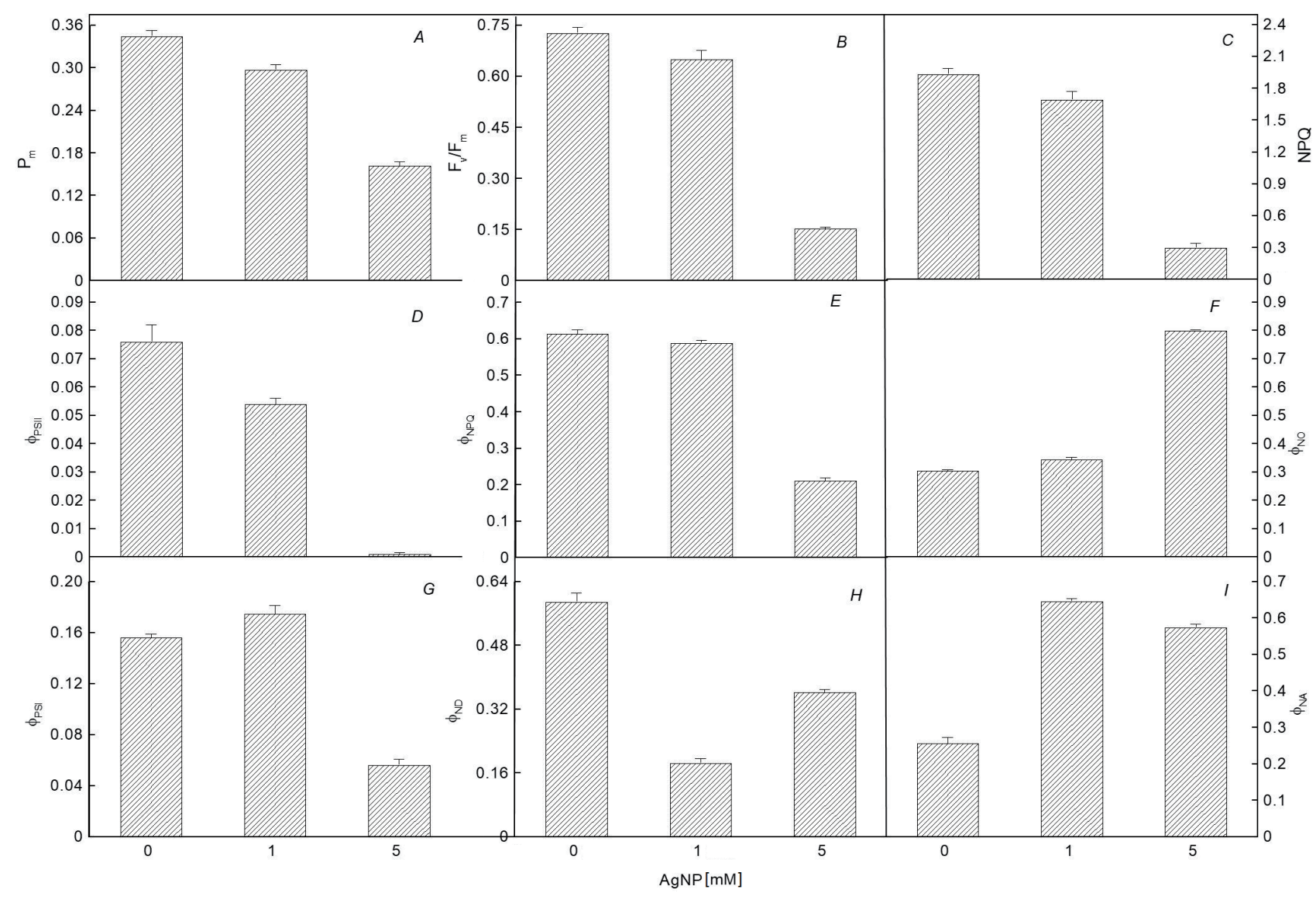

Fig. 4. Impact of AgNPs on photochemistry of wheat plants: $\mathrm{P}_{\mathrm{m}}-$ maximum amplitude of $\mathrm{P} 700$ signal $(A) ; \mathrm{F}_{\mathrm{v}} / \mathrm{F}_{\mathrm{m}}-$ photosynthetic efficiency of PSII under dark adaptation $(B)$; NPQ - nonphotochemical quenching $(C)$; $\Phi_{\text {PSII }}-$ maximum quantum yield of PSII photochemistry $(D)$; $\Phi_{\mathrm{NPQ}}-$ quantum yield of regulated nonphotochemical energy loss in PSII $(E) ; \Phi_{\mathrm{NO}}$ - quantum yield of nonregulated nonphotochemical energy loss in PSII $(F)$; $\Phi_{\mathrm{PSI}}-$ maximum quantum yield of PSI $(G)$; $\Phi_{\mathrm{ND}}-$ quantum yield of regulated nonphotochemical energy loss in PSI $(H) ; \Phi_{\mathrm{NA}}-$ quantum yield of nonregulated nonphotochemical energy loss in PSI $(I)$.

state due to lack of acceptors, was $125 \%$ higher in case of $1 \mathrm{mM}$ AgNPs-treated samples, whereas it was $100 \%$ higher for $5 \mathrm{mM}$ AgNPs-treated samples in comparison with controls (Fig. 4I).

The Chl $a$ fluorescence induction curve with OJIP steps obtained from wheat plants showed a significant change under AgNPs treatment; it was almost linear under $5 \mathrm{mM}$ concentration (Fig. 5A). Some of the important photosynthetic parameters, such as photochemical efficiency $\left(\Phi_{\mathrm{Po}}\right)$, overall photochemical performance index $\left(\mathrm{PI}_{\mathrm{abs}}\right)$, electron transport at PSII acceptor side $\left(\Psi_{\mathrm{ETo}}\right)$, fraction of active PSII reaction centers (RC/ABS), and maximum fluorescence intensity $\left(\mathrm{F}_{\mathrm{m}}\right)$, were calculated according to Strasser (1997), whereas a fraction of active oxygen evolving complex $\left(\mathrm{OEC}_{\mathrm{act}}\right)$ was calculated according to Chen and Cheng (2009) (Fig. 5B). The results indicate a clear impact of AgNPs on P700 redox state and Chl fluorescence in wheat plants.

\section{Discussion}

Our research confirmed previous reports on phytotoxic properties of AgNPs (Rastogi et al. 2017) by showing the reduction in root and shoot growth (Table 1, Fig. 1A). The studied AgNPs were $<100 \mathrm{~nm}$ in diameter, whereas size exclusion limit for plant cell wall has been reported to be in between 5-20 nm (Dietz and Herth 2011), which may restrict its absorption by root cells. It has been previously observed that at high concentrations, the nanoparticles may destroy the cell structure and enter the root cells (Mirzajani et al. 2013), which is also supported by the observation that some of the nanoparticles may induce larger pores in the cell wall (Navarro et al. 2008, Kurepa et al. 2010). Therefore, it can be expected that at high concentrations the studied AgNPs may create an additional pore in the cell wall and may enter the plant. The microscope pictures of roots showed a clear dark spot in roots of the $5 \mathrm{mM}$ AgNPstreated plant (Fig. 1B), which indicates the presence of AgNPs in root cells. At $5 \mathrm{mM}$ AgNPs concentration, the plants were observed to avoid its contact with filter paper, and therefore absorbed the water from the air. Due to plant dependence on atmospheric water, the root hairs were observed to be significantly developed in plants treated with $5 \mathrm{mM}$ AgNPs in comparison with control.

Several studies have shown that the phytotoxic effect on plants is mediated through oxidative burst (Hossain et al. 2015, Rastogi et al. 2017), which may initiate a protective mechanism in plant cells by increasing the production of 


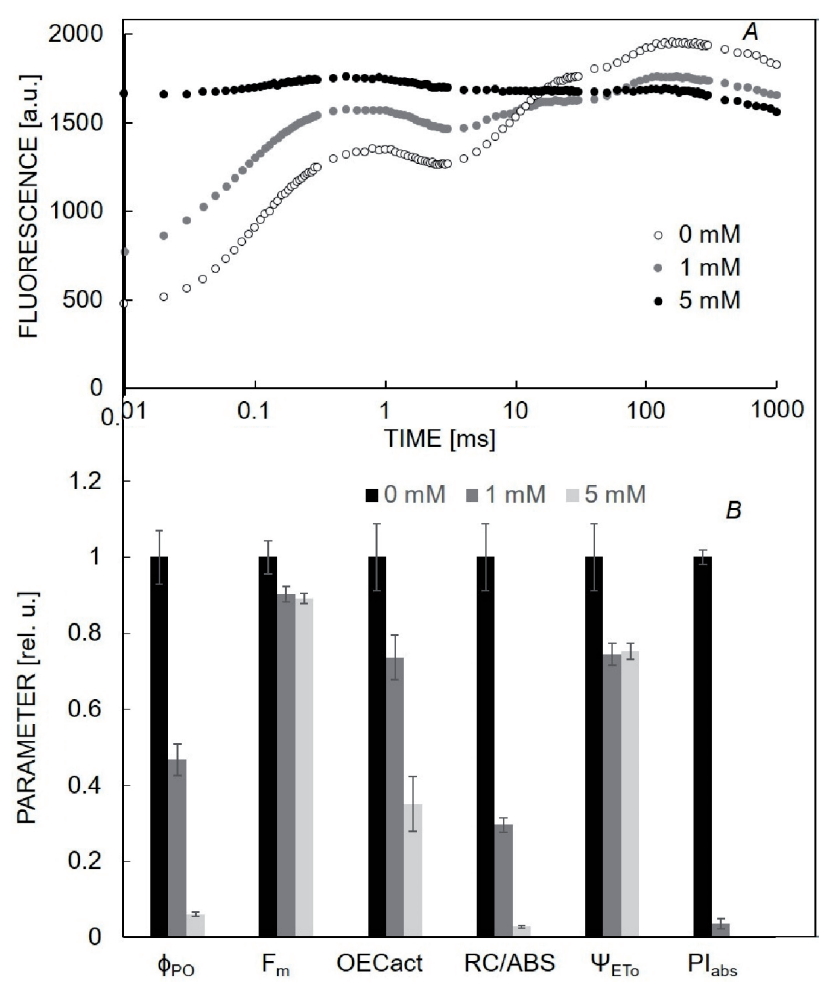

Fig. 5. OJIP test analysis. (A) The fluorescence induction curve and $(B)$ different photosynthetic parameters $\left(\Phi_{\mathrm{P}_{0}}-\right.$ photochemical efficiency; $\mathrm{F}_{\mathrm{m}}$ - maximum fluorescence intensity; $\mathrm{OEC}_{\text {act }}-$ active oxygen evolving complex; RC/ABS - fraction of active PSII reaction centres; $\Psi_{\mathrm{ETO}}-$ electron transport at PSII acceptor side; $\mathrm{PI}_{\text {abs }}$ - overall photochemical performance index) after exposure of wheat to different concentrations of AgNPs.

antioxidants. Catalase is the enzyme known to catalyze the decomposition of hydrogen peroxide to water and oxygen. An increase in catalase activity indicates an increased amount of hydrogen peroxide, whereas, an increased amount of GSH in AgNPs-treated samples indicates a higher production of glutathione (Fig. 2).

Chl concentration is an important indicator for accessing the photosynthesis and growth conditions, whereas flavonoids are molecules which have been detected to be increased under different abiotic stress conditions (Sytar et al. 2015, Schulz et al. 2016, Zivcak et al. 2017). The decrease in the Chl concentration and the increase in flavonoids concentration (Fig. 3) confirmed that studied AgNPs were phytotoxic to wheat.

In addition to other physiological responses, we observed the severe effect of AgNPs on PSI and PSII photochemistry. Whereas the effect of $5 \mathrm{mM}$ AgNPs was evidently lethal, suppressing almost fully the photochemical activities in chloroplasts (Fig. 4), the effect of $1 \mathrm{mM}$ AgNPs was much more interesting. With a decrease in PSII quantum efficiency, a moderate impact of nanoparticles on PSII photochemistry under high light indicated a decrease in electron transport rate by $30 \%$. Typically, under stress conditions, the decrease of $\mathrm{CO}_{2}$ assimilation limits the demand for NADPH and ATP, resulting in a decrease of the rate of linear electron transport, but also in the accumulation of $\mathrm{H}^{+}$in thylakoid lumen. The phenomena result in a significant increase of NPQ and the downregulation of linear electron flow at cytochrome $b_{6} / f$. This is one of the most important photoprotective responses in the chloroplast, which protect the electron transport chain against over-reduction (Joliot and Johnson 2011). However, we observed a decrease in NPQ instead of its increase, which indicates that the regulatory mechanism is not working properly under AgNPs treatment (Fig. $4 C-F$ ). This effect is even more evident when looking at the redox poise of PSI donor $\left(\Phi_{\mathrm{ND}}\right)$ and acceptor $\left(\Phi_{\mathrm{NA}}\right)$ side (Fig. $\left.4 H, I\right)$. It is obvious that PSI acceptor side is highly over-reduced. This state of PSI acceptor side was shown to be responsible for the excessive production of ROS, especially hydroxyl radicals, which may attack sensitive structures in chloroplasts and the whole cell (Takagi et al. 2016). It was clearly shown that, under high light conditions, high level of P700 oxidation (high $\Phi_{\mathrm{ND}}$ ) is crucial for the protection of the PSI acceptor side against over-reduction. However, under conditions of $1 \mathrm{mM}$ AgNPs, the $\Phi_{\mathrm{ND}}$ was observed to be very low. The observed phenomena may be caused by insufficient downregulation of linear electron transport at cytochrome $b_{6} / f$, probably due to low trans-thylakoid proton gradient. A similar effect was observed in mutants with a low PSI content (Brestic et al. 2015) or with a mutation limiting the ability to drive the cyclic electron flow around PSI (Grieco et al. 2012). However, increase in $\Phi_{\mathrm{PSI}}$, in parallel with a decrease in $\Phi_{\text {PSII }}$ suggest that there was an increase of cyclic electron flow due to $1 \mathrm{mM}$ AgNPs treatment. It has been previously reported that, under some specific conditions such as high temperature, excessive leakage of $\mathrm{H}^{+}$through thylakoid membrane can occur (Bukhov et al. 1999). The excessive leakage of $\mathrm{H}^{+}$may lead to low $\Delta \mathrm{pH}$, which results in to over-reduction of PSI acceptor side despite a high cyclic electron flow (Brestic et al. 2016). Thus, we can hypothesize that the AgNPs may cause the leak of $\mathrm{H}^{+}$through thylakoid membrane or proton channels and result in a decrease in $\Delta \mathrm{pH}$ and, hence, the proton motive force, which may negatively influence the ATP synthesis in chloroplasts (Zhang et al. 2009).

One of the direct effects of improper regulation of linear electron transport is the PSI photoinhibition. Our measurements indicate an evident decrease in the content of active PSI unit, indicating PSI photoinhibition. Whereas the PSII photoinihibition is common, PSI in wild type plants is thought to be very resistant to photodamage (Powles 1984), except chilling stress (Sonoike 1996) or fluctuating light (Grieco et al. 2012) conditions. Recently, it was assumed that the photoinhibition of PSI may represent a protective mechanism against oxidative damage of key cell structures under conditions where the linear electron transport cannot be well regulated (Brestic et al. 2016, Tiwari et al. 2016). The nonfunctional PSI centres turn into PSI-NPQs of excitation energy and dissipate thermally the surplus of excitation energy. Photodamage of PSI increases the antenna cross-section of PSI, and conversely decreases that of PSII, through thylakoid protein phosphorylationdependent mechanisms, thus maximizing the amount of excess energy dissipation by PSI. It can represent the 
mechanism of high physiological significance for the survival of plants under specific conditions, in which the flexible mechanisms are not working properly (Tiwari et al. 2016). However, it was shown that the photodamage of PSI may have a high physiological significance, and it indicates a decrease in photosynthetic efficiency and $\mathrm{CO}_{2}$ assimilation capacity, as well as photoprotection of PSII (Zivcak et al. 2015).

Thus, it is obvious that the photochemistry, especially the processes at the level of PSI, is severely affected by the AgNPs treatment. The acceptor site of PSI can be a major source of free radicals (ROS), which are responsible for the oxidative damage observed in this study as well as previously reported by the other authors (Oukarroum et al. 2013, Tripathi et al. 2017, Rastogi et al. 2017). ROS produced at PSI acceptor side can cause damage of other chloroplast structures, such as PSII; this may explain the effects of AgNPs on PSII photochemistry well demonstrated by the analyses of OJIP transient (Fig. 5). In addition to a decrease in $\Phi_{\mathrm{Po}_{\mathrm{o}}}$ and $\mathrm{PI}_{\mathrm{abs}}$, which was evident also from the PAM measurements, we observed several important phenomena related to the status of PSII photochemistry. First, a significant decrease of $\mathrm{RC} / \mathrm{ABS}$ ratio can be attributed mostly to a decrease in the fraction of active PSII RCs. It means that presence of AgNPs leads to a severe inactivation of PSII even at the lower concentration. This can be partly explained by the presence of enhanced K-step in OJIP transient, which indicates a decrease in PSII donor side activity (Strasser 1997). The oxygen evolving complex (OEC) represents one of the sensitive sites, frequently damaged by ROS produced in chloroplasts (Vass 2012). Although the direct effect of AgNPs on OEC cannot be excluded, the indirect effect through ROS produced at the PSI acceptor side is more probable. Interestingly, we observed only a moderate limitation of electron transport at PSII acceptor side $\left(\Psi_{\mathrm{ETO}}\right)$, which is a typical response to many stress factors (Kalaji et al. 2018). The maximum fluorescence signal was still relatively high, which indicates that the decrease in photochemical activity was not associated with a severe molecular degradation of PSII complexes. As the photoinhibition of PSII resulting from a high excitation pressure at PSII acceptor side is typically associated with a decrease in $\mathrm{F}_{\mathrm{m}}$ (Baker and Rosenquist 2004), the observed changes in PSII photochemistry cannot be attributed dominantly to the light-induced PSII damage with a typical turnover of PSII proteins. At least, the repair of damaged PSII is probably not working and inactive PSII are accumulated without recovery. In this respect, we can hypothesize that AgNPs may inhibit the repair of PSII, which may have more serious consequences such as inhibition of PSII activity. Such an effect was also observed under other environmental stresses (Nishiyama et al. 2006). The effect can be caused by the possible insufficient production of ATP needed for PSII repair due to impaired activity of PSI discussed above. Thus, it can be concluded that the studied AgNPs is phytotoxic for wheat seedlings and cause a severe impact on the photosynthetic process, which occurs due to the damage of PSI in the chloroplasts.

\section{References}

Baker N.R., Rosenqvist E.: Applications of chlorophyll fluorescence can improve crop production strategies: an examination of future possibilities. - J. Exp. Bot. 55: 1607-1621, 2004.

Belava V.N., Panyuta O.O., Yakovleva G.M. et al.: The effect of silver and copper nanoparticles on the wheat-Pseudocercosporella herpotrichoides pathosystem. - Nanoscale Res. Lett. 12: 250, 2017.

Boxall P., Purcell J., Wright P.: Human resource management: scope, analysis and significance. - In: Boxall P., Purcell J., Wright P. (ed.): The Oxford Handbook of Human Resource Management. Pp. 1-18. Oxford University Press, Oxford 2007.

Brestic M., Zivcak M., Kunderlikova K. et al.: Low PSI content limits the photoprotection of PSI and PSII in early growth stages of chlorophyll $b$-deficient wheat mutant lines. Photosynth. Res. 125: 151-166, 2015

Brestic M., Zivcak M., Kunderlikova K., Allakhverdiev S.I.: High temperature specifically affects the photoprotective responses of chlorophyll $b$-deficient wheat mutant lines. Photosynth. Res. 130: 251-266, 2016.

Bukhov N.G., Wiese C., Neimanis S., Heber U.: Heat sensitivity of chloroplasts and leaves: leakage of protons from thylakoids and reversible activation of cyclic electron transport. Photosynth. Res. 59: 81-93, 1999.

Chen L.S., Cheng L.: Photosystem 2 is more tolerant to high temperature in apple (Malus domestica Borkh.) leaves than in fruit peel. - Photosynthetica 47: 112-120, 2009.

Dietz K-J., Herth S.: Plant nanotoxicology. - Trends Plant Sci. 16: 582-589, 2011.

Dimkpa C.O., McLean J.E., Martineau N. et al.: Silver nanoparticles disrupt wheat (Triticum aestivum L.) growth in a sand matrix. - Environ. Sci. Technol. 47: 1082-1090, 2013.

El-Temsah Y.S., Joner E.J.: Ecotoxicological effects on earthworms of fresh and aged nano-sized zero-valent iron (nZVI) in soil. - Chemosphere 89: 76-82, 2012.

Grieco M., Tikkanen M., Paakkarinen V. et al.: Steady-state phosphorylation of light-harvesting complex II proteins preserves photosystem I under fluctuating white light. - Plant Physiol. 160: 1896-1910, 2012.

Gruyer N., Dorais M., Bastien C. et al.: Interaction between silver nanoparticles and plant growth. - In: Son J.E., Lee I.B., Oh M.M. (ed.): International Symposium on New Technologies for Environment Control, Energy-Saving and Crop Production in Greenhouse and Plant. Pp. 795-800. ISHS Acta Horticulturae, Jeju, Korea 2014.

Hawthorne J., Musante C., Sinha S.K., White J.C.: Accumulation and phytotoxicity of engineered nanoparticles to Cucurbita pepo. - Int. J. Phytoremediat. 14: 429-442, 2012.

Hossain Z., Mustafa G., Komatsu S.: Plant responses to nanoparticle stress. - Int. J. Mol. Sci. 16: 26644-26653, 2015.

Jasim B., Thomas R., Mathew J., Radhakrishnan E.K.: Plant growth and diosgenin enhancement effect of silver nanoparticles in Fenugreek (Trigonella foenumgraecum L.). Saudi Pharm. J. 25: 443-447, 2017.

Jiang H.S., Qiu X.N., Li G.B. et al.: Silver nanoparticles induced accumulation of reactive oxygen species and alteration of antioxidant systems in the aquatic plant Spirodela polyrhiza. - Environ. Toxicol. Chem. 33: 1398-1405, 2014.

Joliot P., Johnson G.N.: Regulation of cyclic and linear electron flow in higher plants. - P. Natl. Acad. Sci. USA 108: 1331713322, 2011.

Kalaji H.M., Rastogi A., Živčák M. et al.: Prompt chlorophyll fluorescence as a tool for crop phenotyping: an example of barley landraces exposed to various abiotic stress factors. - 
Photosynthetica 56: 953-961, 2018.

Kalaji H.M., Schansker G., Ladle R.J. et al.: Frequently asked questions about in vivo chlorophyll fluorescence: practical issues. - Photosynth. Res. 122: 121-158, 2014.

Khaydarov R.R., Khaydarov R.A., Gapurova O. et al.: Antimicrobial effects of silver nanoparticles synthesized by an electrochemical method. - In Reithmaier J.P., Petkov P., Kulisch W., Popov C. (ed.): Nanostructured Material for Advanced Technological Applications. NATO Science for Peace and Security Series B: Physics and Biophysics. Pp. 215-218. Springer, Dordrecht 2009.

Kurepa J., Paunesku T., Vogt S. et al.: Uptake and distribution of ultrasmall anatase $\mathrm{TiO}_{2}$ Alizarin red S nanoconjugates in Arabidopsis thaliana. - Nano Lett. 10: 2296-2302, 2010.

Mirzajani F., Askari H., Hamzelou S. et al.: Effect of silver nanoparticles on Oryza sativa L. and its rhizosphere bacteria. - Ecotoxicol. Environ. Safe. 88: 48-54, 2013.

Navarro E., Piccapietra F., Wagner B. et al.: Toxicity of silver nanoparticles to Chlamydomonas reinhardtii. - Environ. Sci. Technol. 42: 8959-8964, 2008.

Nishiyama Y., Allakhverdiev S.I., Murata N.: A new paradigm for the action of reactiv e oxygen species in the photoinhibition of photosystem II. - BBA- Bioenergetics. 1757: 742-749, 2006.

Oukarroum A., Barhoumi L., Pirastru L., Dewez D.: Silver nanoparticle toxicity effect on growth and cellular viability of the aquatic plant Lemna gibba. - Environ. Toxicol. Chem. 32: 902-907, 2013

Pallavi, Mehta C.M., Srivastava R. et al.: Impact assessment of silver nanoparticles on plant growth and soil bacterial diversity. -3 Biotech. 6: 254, 2016.

Perreault F., Samadani M., Dewez D.: Effect of soluble copper released from copper oxide nanoparticles solubilisation on growth and photosynthetic processes of Lemna gibba L. Nanotoxicology. 8: 374-382, 2014.

Pokhrel L.R., Dubey B.: Evaluation of developmental responses of two crop plants exposed to silver and zinc oxide nanoparticles. - Sci. Total Environ. 452-453: 321-332, 2013.

Powles S.B.: Photoinhibition of photosynthesis induced by visible light. - Annu. Rev. Plant Physio. 35: 15-44, 1984.

Qian H., Peng X., Han X. et al.: Comparison of the toxicity of silver nanoparticles and silver ions on the growth of terrestrial plant model Arabidopsis thaliana. - J. Environ. Sci.-China 25: 1947-1955, 2013.

Rai M., Yadav A., Gade A.: Silver nanoparticles as a new generation of antimicrobials. - Biotechnol. Adv. 27: 76-83, 2009.

Rastogi A., Zivcak M., Sytar O. et al.: Impact of metal and metal oxide nanoparticles on plant: A critical review. - Front. Chem. 5: 78, 2017.

Santos C.S.C., Gabriel B., Blanchy M. et al.: Industrial applications of nanoparticles - A prospective overview. - Mater. Today-Proc. 2: 456-465, 2015.

Schulz E., Tohge T., Zuther E. et al.: Flavonoids are determinants of freezing tolerance and cold acclimation in Arabidopsis thaliana. - Sci. Rep. 6: 34027, 2016.

Sonoike K.: Photoinhibition of photosystem I: Its physiological significance in the chilling sensitivity of plants. - Plant Cell Physiol. 37: 239-247, 1996.

Srivastava A., Strasser R.J., Govindjee: Greening of peas: parallel measurements of $77 \mathrm{~K}$ emission spectra, OJIP chlorophyll $a$ fluorescence transient, period four oscillation of the initial fluorescence level, delayed light emission and $\mathrm{P} 700^{*}$. Photosynthetica 37: 365-392, 1999.

Strasser B.J., Strasser R.J.: Measuring fast fluorescence transients to address environmental questions: The JIP-test. - In: Mathi
P. (ed.): Photosynthesis: from Light to Biosphere. Pp. 977980. Kluwer Academic Publishers, Dordrecht 1995.

Strasser R.J., Tsimilli-Michael M., Qiang S., Goltsev V.: Simultaneous in vivo recording of prompt and delayed fluorescence and $820-\mathrm{nm}$ reflection changes during drying and after rehydration of the resurrection plant Haberlea rhodopensis. Biochim. Biophys. Acta 1797: 1313-1326, 2010.

Strasser R.J., Tsimilli-Michael M., Dangre D., Rai M.: Biophysical phenomics reveals functional building blocks of plants system biology: a case study for evaluation of the impast of Mycorrhization with Piriformospora indica. - In: Varma A., Oelmüller R. (ed.): Advanced Techniques in Soil Biology. Pp. 319-338. Springer, Dordrecht 2004.

Strasser R.J., Srivastava A., Tsimilli-Michael M.: The fluorescence transient as a tool to characterize and screen photosynthetic samples. - In: Yunus M., Pathre U., Mohanty P. (ed.): Probing Photosynthesis: Mechanisms, Regulation and Adaptation. Pp. 445-483. Taylor \& Francis, London 2000.

Strasser B.J.: Donor side capacity of photosystem II probed by chlorophyll $a$ fluorescence transient. - Photosynth. Res. 52: 147-155, 1997.

Sytar O., Bruckova K., Hunkova E. et al.: The application of multiplex fluorimetric sensor for the analysis of flavonoids content in the medicinal herbs family Asteraceae, Lamiaceae, Rosaceae. - Biol. Res. 48: 5, 2015.

Takagi D., Takumi S., Hashiguchi M. et al.: Superoxide and singlet oxygen produced within the thylakoid membranes both cause photosystem I photoinhibition. - Plant Physiol. 171: 1626-1634, 2016.

Tiwari A., Mamedov F., Grieco M. et al.: Photodamage of ironsulphur clusters in photosystem I induces non-photochemical energy dissipation. - Nat. Plants 2: 16035, 2016.

Tripathi D.K., Singh S., Singh S. et al.: Nitric oxide alleviates silver nanoparticles (AgNps)-induced phytotoxicity in Pisum sativum seedlings. - Plant Physiol. Bioch. 110: 167-177, 2017.

Vass I.: Molecular mechanisms of photodamage in the Photosystem II complex. - BBA-Bioenergetics 1817: 209217, 2012.

Vinković T., Novák O., Strnad M. et al.: Cytokinin response in pepper plants (Capsicum annuum L.) exposed to silver nanoparticles. - Environ. Res. 156: 10-18, 2017.

Wijnhoven S.W.P., Peijnenburg W.J.G.M., Herberts C.A. et al.: Nano-silver - A review of available data and knowledge gaps in human and environmental risk assessment. - Nanotoxicology 3: 109-138, 2009.

Yin L., Colman B.P., McGill B.M. et al.: Effects of silver nanoparticle exposure on germination and early growth of eleven wetland plans. - PLoS ONE 7: e47674, 2012.

Zhang R., Cruz J.A., Kramer D.M. et al.: Moderate heat stress reduces the $\mathrm{pH}$ component of the transthylakoid proton motive force in light-adapted, intact tobacco leaves. - Plant Cell Environ. 32: 1538-1547, 2009.

Zivcak M., Brestic M., Kunderlikova K. et al.: Repetitive light pulse-induced photoinhibition of photosystem I severely affects $\mathrm{CO}_{2}$ assimilation and photoprotection in wheat leaves. - Photosynth. Res. 126: 449-463, 2015.

Zivcak M., Brückova K., Sytar O. et al.: Lettuce flavonoids screening and phenotyping by chlorophyll fluorescence excitation ratio. - Planta 245: 1215-1229, 2017.

Zuverza-Mena N., Armendariz R., Peralta-Videa J.R., GardeaTorresdey J.L.: Effects of silver nanoparticles on radish sprouts: Root growth reduction and modifications in the nutritional value. - Front Plant Sci. 7: 90, 2016.

(C) The authors. This is an open access article distributed under the terms of the Creative Commons BY-NC-ND Licence. 